\title{
Multi-mode Damage Detection Methods with Piezoelectric Wafer Active Sensors
}

\author{
LINGYU YU* AND Victor GIURGIUTIU \\ Department of Mechanical Engineering, University of South Carolina, Columbia, SC 29208, USA
}

\begin{abstract}
PWAS are small and non-invasive transducers that can be easily affixed to a structure to interrogate it at will. Their wideband non-resonant properties make them advantageous candidates for multiple modes structural health monitoring (SHM) and nondestructive evaluation (NDE) simply by changing the driving frequencies. This paper presents research results obtained by using PWAS transducer for SHM/NDE. The results are given in the following directions: (1) PWAS operation principles; (2) PWAS electromechanical impedance measurements; (3) PWAS ultrasonic SHM/NDE; and (4) PWAS multi-mode corrosion detection. The analytical methods and experimental results can be successfully used in the design of active SHM systems to be embedded on operational structures, such as aircraft panels.
\end{abstract}

Key Words: PWAS, piezoelectric, multiple modes, impedance, Lamb waves, phased array, damage detection, SHM, NDE.

\section{INTRODUCTION}

$\mathbf{S}_{\mathrm{og}}^{\mathrm{T}}$ TRUCTURAL health monitoring (SHM) is a technology to collect data about critical structural components using embedded sensing elements to provide detection and diagnosis when damage occurs. Common forms of structural damage include holes, cracks, corrosion, etc. One method of high efficient SHM of thin-wall structures is to utilize guided waves, e.g., Lamb waves in thin plates (Rose, 1999). Guided waves can propagate inside thin-wall plates and shallow shells, traveling large distances with very little amplitude loss. Traditionally, guided waves have been generated by a relatively large ultrasonic transducer (Alleyne and Cawley, 1992) or by a comb transducer (Rose et al., 1998). Although Lamb wave phased-array methods are promising, the fact that they utilize conventional ultrasonic transducers, which are bulky and expensive, may make them unsuitable for SHM. The advent of commercially available low-cost piezoelectric wafer active sensors, namely PWAS, has opened new opportunities for in situ ultrasonic testing as needed during the SHM processes. The PWAS have the potential for in situ ultrasonics through inexpensive and unobtrusive deployment. Early work has shown that PWAS devices can successfully be used for SHM applications, i.e., Figure 1 (Lin and Yuan, 2001; Ihn and Chang, 2002; Raghavan and Cesnik, 2005; Nieuwenhuis et al., 2005; Han et al., 2006). Giurgiutiu and collaborators (2005)

*Author to whom correspondence should be addressed. E-mail: yu3@engr.sc.edu

Figures $1-6$ and $9-16$ appear in color online: http://jim.sagepub.com have shown that PWAS can be used as both active and passive probes and thus can address four SHM needs (Figure 1):

1. Active sensing of far-field damage using pulse-echo, pitch-catch, and phased-array methods.

2. Active sensing of near-field damage using highfrequency impedance method.

3. Passive sensing of crack initiation and growth through acoustic emission.

4. Passive sensing of damage-generating events through detection of low-velocity impacts.

This paper will present multiple modes in situ SHM methods using PWAS transducers with impedance, pitchcatch, and/or pulse-echo methods for damage detection (cracks and corrosion) in metallic plate structures. Principles of PWAS transducers for impedance measurement and ultrasonic inspection are given first. Then several examples of using the various PWAS methods alone or combined are provided to show the application significance.

\section{PIEZOELECTRIC WAFER ACTIVE SENSORS PRINCIPLES}

This section addresses the behavior of a bonded (constrained) PWAS when excited by an alternating electric voltage. As illustrated in Figure 2(a), PWAS are small and unobtrusive. A $5 \times 5 \times 0.2 \mathrm{~mm}^{3}$ square PWAS weighs about $50 \mathrm{mg}$ and costs about $\$ 10$. 
(a)

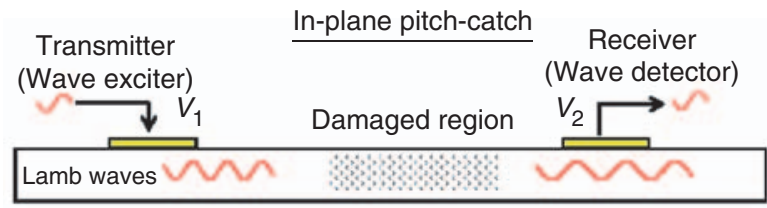

(b) Transmitter-receiver In-plane pulse-echo

PWAS
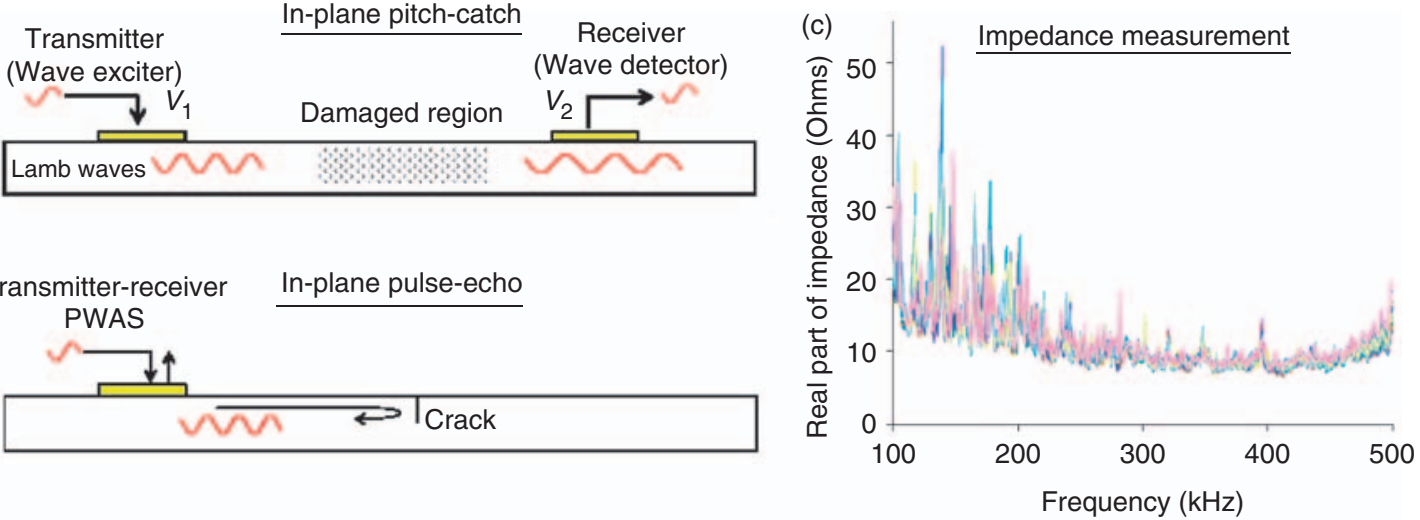

(d) Transmitter-receiver Thickness pulse-echo PWAS

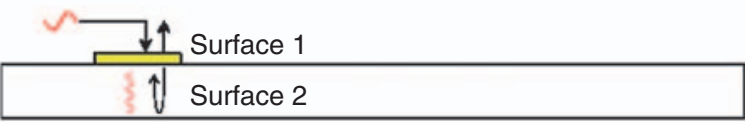

Figure 1. PWAS SHM/NDE applications: (a) pitch-catch method in plane motion; (b) pulse-echo method in plane motion; (c) impedance measurement; and (d) pulse-echo method in thickness mode.

(a)

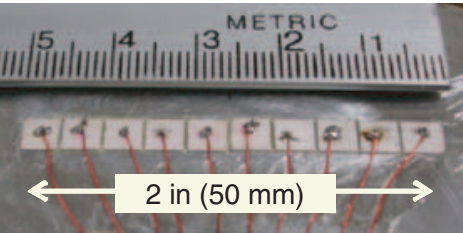

(b)

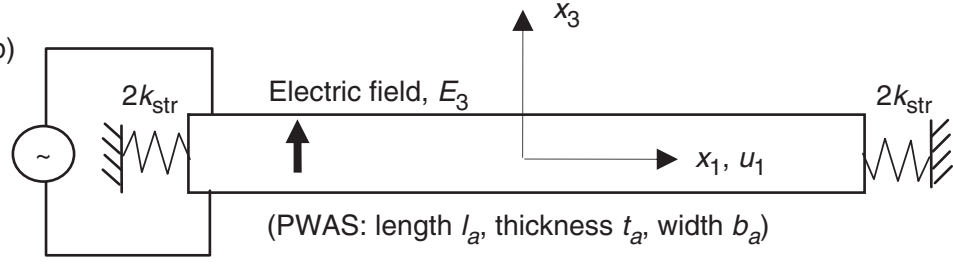

$V(t)=V_{0} \sin (t)$

Figure 2. Embedded piezoelectric wafer active sensors: (a) a phased array composed of ten $5 \mathrm{~mm}$ square PWAS permanently attached to the host structure; (b) a 1D bonded PWAS under electric excitation, constrained by structural stiffness $k_{\text {str }}$.

They utilize the coupling between in-plane strain and transverse electric field.

\section{D PWAS Analysis}

PWAS operate on the piezoelectric principle that couples the electrical and mechanical variables (mechanical strain $S_{1}$, mechanical stress $T_{1}$, electrical field $E_{3}$, and electrical displacement $D_{3}$ ) in the material in the one dimensional form:

$$
\begin{aligned}
& S_{1}=s_{11}^{E} T_{1}+d_{31} E_{3} \\
& D_{3}=d_{31} T_{1}+\varepsilon_{33}^{T} E_{3}
\end{aligned}
$$

where, $s_{11}^{E}$ is the mechanical compliance of the material at zero electrical field $\left(E_{3}=0\right), T_{1}$ is the stress, $\varepsilon_{33}^{T}$ is the dielectric constant at zero stress $\left(T_{1}=0\right)$, and $d_{31}$ is the induced strain coefficient (mechanical strain per unit electric field). PWAS utilize the $d_{31}$ coupling between in-plane strain and transverse electric field.

Consider a PWAS of length $l_{a}$, width $b_{a}$, and thickness $t_{a}$ that is undergoing longitudinal expansion $\left(u_{1}\right)$ induced by the thickness polarization electric field $\left(E_{3}\right)$, as shown in Figure 2b. The electric field is produced by the application of a harmonic voltage, $V(t)=\hat{V} e^{j \omega t}$, between the top and bottom surfaces (electrodes). The resulting electric field $E_{3}=V / t$, is assumed uniform over the PWAS. Assume that $h, b$, and $l$ have widely separated values $(h \ll b \ll l)$ such that the length, width, and thickness motions are practically uncoupled. The motion predominantly in the longitudinal direction $\left(x_{1}\right)$ will be considered (1D assumption). When PWAS is bonded to the structure, the structure will constrain the PWAS motion with a structural stiffness $\left(k_{\text {str }}\right)$; that is to say, PWAS are elastically constrained as shown in Figure 2b. In this model, the overall structural stiffness applied to the PWAS is split into two equal components applied to the PWAS ends, $2 k_{\text {str }}$ each, such that:

$$
k_{\text {total }}=\left[\left(2 k_{\text {str }}\right)^{-1}+\left(2 k_{\text {str }}\right)^{-1}\right]^{-1}=k_{\text {str }} .
$$

Note that the effective structural stiffness, $k_{\text {str }}$, is a frequency-dependent complex quantity reflecting the structural dynamics. The boundary conditions applied 
at the PWAS ends balance the resulting stress $\left(T_{1} b_{a} t_{a}\right)$ with the spring reaction force $\left(2 k_{\mathrm{str}} u_{1}\right)$ :

$$
\begin{array}{r}
T_{1}\left(\frac{l_{a}}{2}\right) b_{a} t_{a}=-2 k_{\mathrm{str}} u_{1}\left(\frac{l_{a}}{2}\right), \\
T_{1}\left(-\frac{l_{a}}{2}\right) b_{a} t_{a}=2 k_{\mathrm{str}} u_{1}\left(-\frac{l_{a}}{2}\right) .
\end{array}
$$

Using the Newton's law of motion $\left(T_{1}^{\prime}=\rho \ddot{u}_{1}\right)$ and the strain-displacement relation $\left(S_{1}=u_{1}^{\prime}\right)$, the axial wave equation can be obtained:

$$
\ddot{u}_{1}=c_{a}^{2} u_{1}^{\prime \prime} .
$$

By introducing the notations: induced strain $S_{\mathrm{ISA}}=d_{31} \hat{E}_{3}, \quad$ displacement $u_{\mathrm{ISA}}=S_{\mathrm{ISA}} l=\left(d_{31} \hat{E}_{3}\right) l$; wavenumber $\gamma, \gamma=\omega / c$; wavelength $\lambda, \lambda=c T=c / f$, $f=\omega / 2 \pi$, quasi-static PWAS stiffness $k_{\mathrm{PWAS}}=A_{a} / s_{11}^{E} l_{a}$, piezoelectric material wave speed $c_{a}^{2}=1 / \rho s_{11}^{E}$, and $\left(\partial / \partial x_{1}\right)()=()^{\prime}, \quad(\partial / \partial t)()=(), \quad$ the general solution $u_{1}(x, t)=\hat{u}(x) e^{j \omega t}$ of Equation (5) can be obtained as:

$$
\hat{u}(x)=\frac{1}{2} u_{\mathrm{ISA}} \frac{\sin \gamma x}{\gamma l \cos (\gamma l / 2) / 2+\gamma \sin (\gamma l / 2)} .
$$

If the system determinant of the boundary conditions is nonzero $(\Delta \neq 0)$ (Giurgiutiu and Lyshevski, 2003):

$$
\begin{aligned}
\Delta & =\left|\begin{array}{cc}
\phi \cos \phi+r \sin \phi & -(\phi \sin \phi-r \cos \phi) \\
\phi \cos \phi+r \sin \phi & (\phi \sin \phi-r \cos \phi)
\end{array}\right| \\
& =2(\phi \cos \phi+r \sin \phi)(\phi \sin \phi-r \cos \phi) .
\end{aligned}
$$

The electrical responses of PWAS under harmonic electric excitation can be represented as the admittance $(Y)$ that is defined as the ratio between the current and the voltage and the impedance $(Z)$ that is the inverse of admittance (Giurgiutiu and Lyshevski, 2003):

$$
\begin{aligned}
& Y=\frac{\hat{I}}{\hat{V}}=j \omega C\left[1-k_{31}^{2}\left(1-\frac{1}{r+\phi \cot \phi}\right)\right] \\
& Z=\frac{\hat{V}}{\hat{I}}=\frac{1}{j \omega C}\left[1-k_{31}^{2}\left(1-\frac{1}{r+\phi \cot \phi}\right)\right]^{-1}
\end{aligned}
$$

with $\phi=\gamma l / 2, \quad k_{31}^{2}=d_{31}^{2} /\left(s_{11}^{E} \varepsilon_{33}^{T}\right) \quad$ [electromechanical (E/M) coupling coefficient], $C=\varepsilon_{33}^{T} b_{a} l_{a} / t_{a}$ (stress-free PWAS capacitance), and $r$ as the structural stiffness ratio. For free PWAS, $r=0\left(k_{\mathrm{str}}=0\right)$, while for full constrained PWAS, $r \rightarrow 8\left(k_{\mathrm{str}} \rightarrow 8\right)$. When the oscillation frequency is so low that the dynamic effects inside the PWAS are negligible, quasi-static conditions are encountered with $\phi=0$, i.e., $\gamma l=0$.
The determinant in Equation (7) is zero when the first or second parenthesis is zero, corresponding to two types of PWAS resonances, mechanical resonances $(\phi \sin \phi-r \cos \phi=0)$ or $\mathrm{E} / \mathrm{M}$ resonances $(\phi \cos \phi+r \sin \phi=0)$. The $\mathrm{E} / \mathrm{M}$ resonances are specific to piezoelectric materials. They reflect the coupling between the mechanical and electrical variables. During the $\mathrm{E} / \mathrm{M}$ resonances, two conditions may happen:

- Resonance, when $Y \rightarrow \infty$; that is, $Z=0$. This resonance is associated with the situation in which a device is drawing very large current when excited harmonically with a constant voltage at a given frequency.

- Anti-resonance, when $Y=0$; that is, $Z \rightarrow \infty$. This anti-resonance is associated with the situation in which a device under constant voltage excitation draws almost no current.

\section{D Circular PWAS}

The 2D constrained PWAS behavior can be analyzed using constitutive equation in the cylindrical coordinates $(r, \theta, z)$ (Figure 3):

$$
\begin{aligned}
& S_{r r}=s_{11}^{E} T_{r r}+s_{12}^{E} T_{\theta \theta}+d_{31} E_{z} \\
& S_{\theta \theta}=s_{12}^{E} T_{r r}+s_{11}^{E} T_{\theta \theta}+d_{31} E_{z} \\
& D_{z}=d_{31}\left(T_{r r}+T_{\theta \theta}\right)+\varepsilon_{33}^{T} E_{z} .
\end{aligned}
$$

The wave equation in polar coordinates is:

$$
\frac{\partial^{2} u_{r}}{\partial r^{2}}+\frac{1}{r} \frac{\partial u_{r}}{\partial r}-\frac{u_{r}}{r^{2}}=\frac{1}{c_{p}} \frac{\partial^{2} u_{r}}{\partial t^{2}}
$$

where, $c_{p}=\sqrt{1 /\left[\rho s_{11}^{E}\left(1-v_{a}^{2}\right)\right]}, \quad v_{a}^{2}=s_{12}^{E} / s_{11}^{E}$. Equation (13) provides a general solution in terms of Bessel functions of the first kind $\left(J_{1}\right)$, in the form:

$$
u_{r}(r, t)=A J_{1}\left(\frac{w r}{c}\right) e^{j \omega t}
$$

where, the coefficient $A$ is determined from the boundary conditions. When PWAS is bonded to a structure, at the boundary $r=r_{a}$, the boundary conditions is:

$$
T_{r r}\left(r_{a}\right) t_{a}=k_{\mathrm{str}}(\omega) u_{r}\left(r_{a}\right) .
$$

The electric impedance is calculated as the ratio between voltage and current amplitudes:

$$
\begin{aligned}
Z(\omega) & =\left\{j \omega C\left(1-k_{p}^{2}\right)\right. \\
& {\left.\left[1+\frac{k_{p}^{2}}{1-k_{p}^{2}} \frac{\left(1+v_{a}\right) J_{1}\left(\phi_{a}\right)}{\phi_{a} J_{0}\left(\phi_{a}\right)-\left(1-v_{a}\right) J_{1}\left(\phi_{a}\right)-\chi(\omega)\left(1+v_{a}\right) J_{1}\left(\phi_{a}\right)}\right]\right\}^{-1} }
\end{aligned}
$$



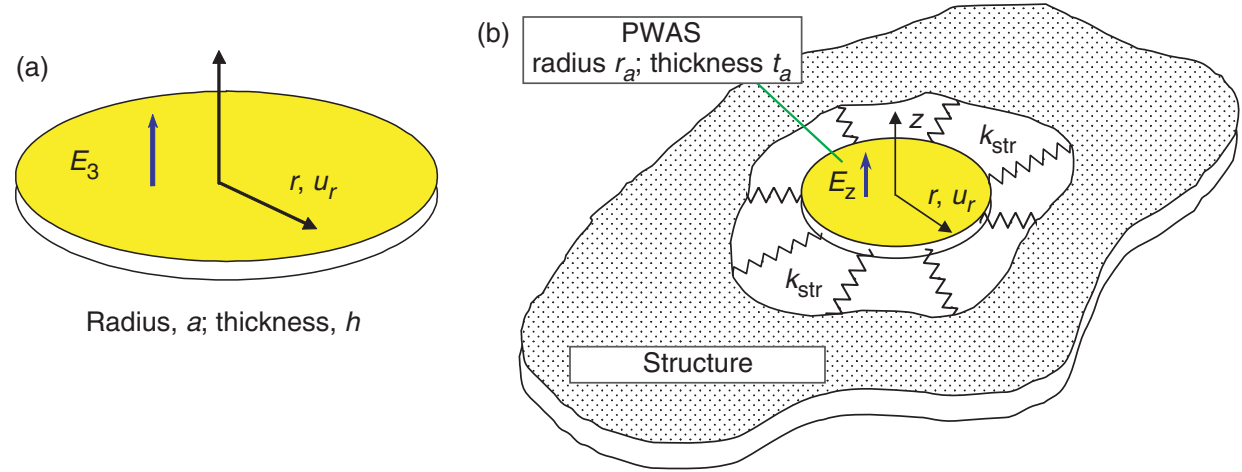

Figure 3. Circular PWAS illustration: (a) the schematic; (b) a circular PWAS constrained by structural stiffness, $k_{\text {str }}(\omega)$.
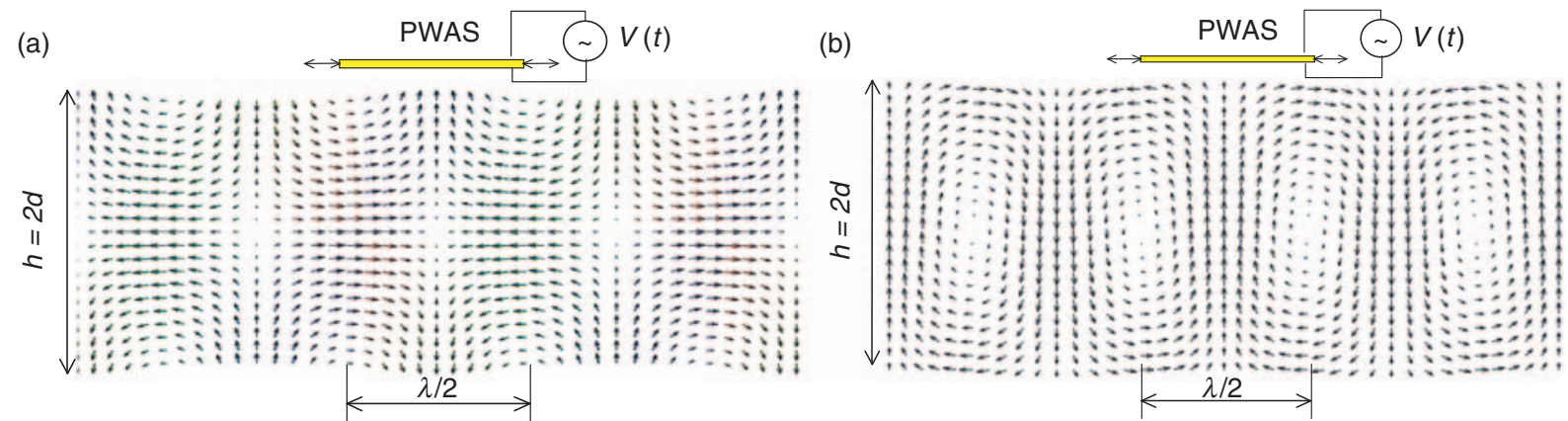

Figure 4. PWAS interaction with Lamb waves in a plate: (a) $S_{0}$ mode; (b) $A_{0}$ mode (Giurgiutiu and Cuc, 2005).

with $\quad \phi_{a}=\omega r_{a} / c, \quad \chi(\omega)=\left(k_{\mathrm{str}}(\omega) / k_{\mathrm{PWAS}}\right) \quad($ dynamic stiffness factor), $\quad k_{p}^{2}=2 d_{31}^{2} /\left[s_{11}^{E}\left(1-v_{a}\right) \varepsilon_{33}^{T}\right] \quad$ (planar coupling factor). Equation (16) predicts that the $\mathrm{E} / \mathrm{M}$ impedance spectrum can be measured by the impedance analyzer at the embedded PWAS terminals during a structural health monitoring process and it allows for direct comparison between calculated predictions and experimental results. The structural dynamic is reflected in Equation (16) through the dynamic stiffness factor $\chi(\omega)$, which contains the dynamic stiffness of the structure.

\section{PWAS ULTRASONIC TRANSDUCERS}

For embedded non-destructive evaluation (NDE) applications, PWAS can be used as embedded ultrasonic transducers. PWAS act as both Lamb wave exciters and detectors (Figure 4). PWAS couple their in-plane motion with the particle motion of Lamb waves on the material surface. The in-plane PWAS motion is excited by the applied oscillatory voltage through the $d_{31}$ piezoelectric coupling. Circular PWAS excite omnidirectional Lamb waves that propagate in circular wave fronts. Omnidirectional Lamb waves are also generated by square PWAS, though their pattern is somewhat irregular in proximity to the PWAS. At a far enough distance $(r \gg a)$, the wave front generated by square PWAS is practically identical with that generated by circular PWAS. The PWAS ultrasonic transducer operation is fundamentally different from that of conventional ultrasonic probes, in that:

1. PWAS achieve Lamb wave excitation and sensing through surface 'pinching' (in-plane strains), while conventional ultrasonic probes excite through surface 'tapping' (normal stress).

2. PWAS are strongly coupled with the structure and follow the structural dynamics, while conventional ultrasonic probes are relatively free from the structure and follow their own dynamics.

3. PWAS are non-resonant wideband devices, while conventional ultrasonic probes are narrowband resonators.

\section{Shear-layer Interaction between PWAS and the Host Structure}

The transmission of actuation and sensing between the PWAS and the structure is achieved through the adhesive layer. The adhesive layer acts as a shear layer, in which the mechanical effects are transmitted through shear effects. As shown in Figure 5(a), a thin-wall structure of thickness $t$ and elastic modulus $E$ is attached with a PWAS of thickness $t_{a}$ and elastic modulus $E_{a}$ to the upper surface through a bonding layer of thickness $t_{b}$ and shear modulus $G_{b}$. The PWAS length is $l_{a}$ while the halflength is $a=l_{a} / 2$. In addition, $t=2 d$. The $1 \mathrm{D}$ plane-strain 
(a)

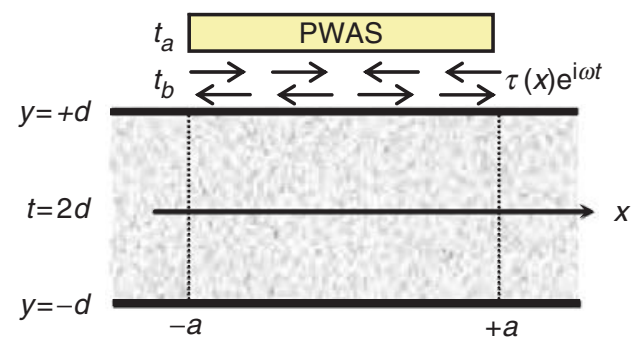

(b)

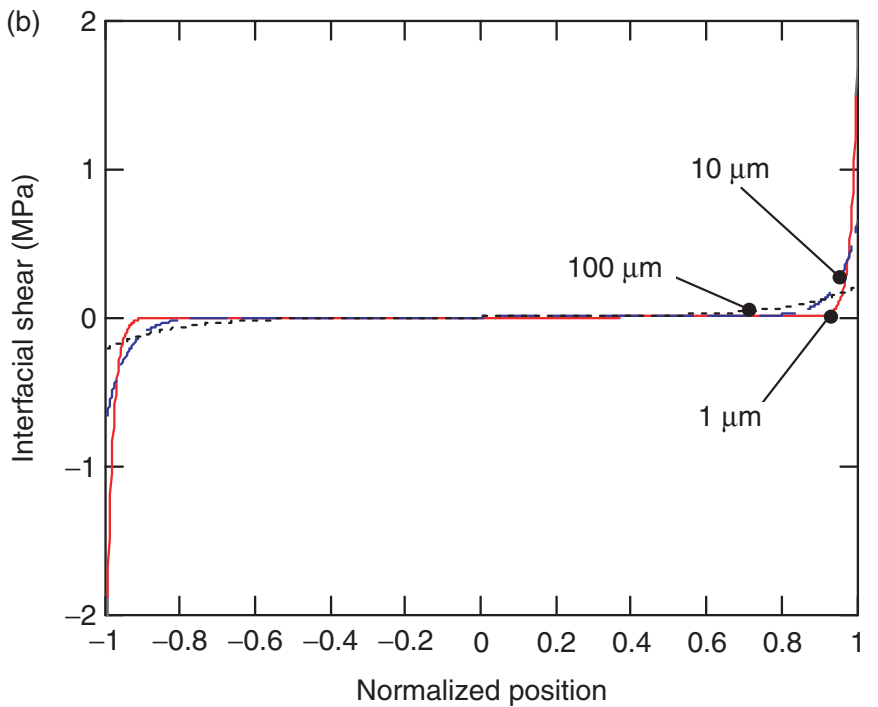

Figure 5. Shear-lag interaction between PWAS and structure: (a) interaction between the PWAS and the structure; (b) variation of shear-lag transfer mechanism with bond thickness.

analysis was an extension of the shear-lag analysis of PWAS excitation of thin-wall structures pioneered by Crawley and de Luis (1987) for static morphing and the quasi-static low-frequency vibrations. Upon application of an electric voltage, the PWAS experiences an induced strain of $\varepsilon_{\text {ISA }}=d_{31} V / t$. The induced strain is transmitted to the structure through the boding layer interfacial shear stress $(\tau)$. The effect of the PWAS is transmitted to the structure through the interfacial shear stress of the bonding layer. A small shear stress value in the bonding layer produces a gradual transfer of strain from the PWAS to the structure, whereas a large shear stress produces a rapid transfer. Because the PWAS ends are stress-free, the build up of strain takes place at the ends, and it is more rapid when the shear stress is more intense. For large value of shear-lag parameter (known as $\Gamma a)$, the shear transfer process becomes concentrated towards the PWAS ends. Figure $5 \mathrm{~b}$ presents the results of the simulations on an APC-PZT-850 PWAS $\left(E_{a}=\right.$ $\left.63 \mathrm{GPa}, \quad t_{a}=0.2 \mathrm{~mm}, \quad l_{a}=7 \mathrm{~mm}, \quad d_{31}=-175 \mathrm{~mm} / \mathrm{kV}\right)$ bonded to a thin-wall aluminum structure $(E=70 \mathrm{GPa}$ and $t=1 \mathrm{~mm})$ using cynoacrylate adhesive $\left(G_{b}=2 \mathrm{GPa}\right)$ of various thickness, $t_{b}=1,10,100 \mu \mathrm{m}$. It reveals that a relatively thick bonding layer produces a low $\Gamma a$ value $-\mathrm{a}$ slow transfer over the entire span of the PWAS (the $100 \mu \mathrm{m}$ curves) - whereas a very thin bonding layer produces a very rapid transfer (the $1 \mu \mathrm{m}$ curves) that is confined to the ends.

The shear lag analysis indicates that in the limit, as $\Gamma a \rightarrow \infty$, all the load transfer can be assumed to take place at the PWAS actuator ends. This leads to the concept of ideal bonding, also known as the pin-force model, in which all the load transfer takes place over an infinitesimal region at the PWAS ends, and the induced strain action is assumed to consist of a pair of concentrated forces applied at the ends. Using the shear-lag model, the energy transferred from PWAS to the structure can be found by analyzing either the elastic energy in the structure or work done by the shear stresses at the structural surface.

\section{Tuned PWAS Lamb Waves Generation}

The excitation of Lamb waves with PWAS is studied by considering the excitation applied by the PWAS through a surface stress $\tau=\tau_{0}(x) e^{j \omega t}$ applied to the upper surface of a plate in the form of shear-lag adhesion stresses over the $(-a,+a)$ interval. Applying a space domain Fourier transform analysis of the basic Lamb wave equations to yield the strain wave and displacement wave solutions (Giurgiutiu, 2005):

$$
\begin{aligned}
\left.\varepsilon_{x}(x, t)\right|_{y=d}=-i \frac{a \tau_{0}}{\mu}[ & \sum_{\xi^{S}} \sin \left(\xi^{S} a\right) \frac{N_{S}\left(\xi^{S}\right)}{D_{S}^{\prime}\left(\xi^{S}\right)} e^{i\left(\xi^{S} x-\omega t\right)} \\
& \left.+\sum_{\xi^{A}} \sin \left(\xi^{A} a\right) \frac{N_{A}\left(\xi^{A}\right)}{D_{A}^{\prime}\left(\xi^{A}\right)} e^{i\left(\xi^{A} x-\omega t\right)}\right]
\end{aligned}
$$

$$
\begin{aligned}
& N_{S}=\xi \beta\left(\xi^{2}+\beta^{2}\right) \cos (\alpha d) \cos (\beta d), \\
& D_{S}=\left(\xi^{2}-\beta^{2}\right)^{2} \cos (\alpha d) \sin (\beta d)+4 \xi^{2} \alpha \beta \sin (\alpha d) \cos (\beta d) \\
& N_{A}=\xi \beta\left(\xi^{2}+\beta^{2}\right) \sin (\alpha d) \sin (\beta d), \\
& D_{A}=\left(\xi^{2}-\beta^{2}\right)^{2} \sin (\alpha d) \cos (\beta d)+4 \xi^{2} \alpha \beta \cos (\alpha d) \sin (\beta d)
\end{aligned}
$$

where, $\xi^{S}$ and $\xi^{A}$ are the zeros of $D_{S}$ and $D_{A}$, respectively. We can note that these are the solutions of the Rayleigh-Lamb equation. Raghavan and Cesnik (2005) extended these results to the case of a circular transducer coupled with circular-crested Lamb waves 
and proposed corresponding tuning prediction formulae based on Bessel functions:

$$
\begin{aligned}
\left.\varepsilon_{r}(r, t)\right|_{z=d}= & \pi \frac{\tau_{0} a}{\mu} e^{i \omega t}\left[\sum_{\xi^{S}} J_{1}\left(\xi^{S} a\right) \xi^{S} \frac{N_{S}\left(\xi^{S}\right)}{D_{S}^{\prime}\left(\xi^{S}\right)} H_{1}^{(2)}\left(\xi^{S} r\right)\right. \\
& \left.+\sum_{\xi_{A}} J_{1}\left(\xi^{A} a\right) \xi^{A} \frac{N_{A}\left(\xi^{A}\right)}{D_{A}^{\prime}\left(\xi^{A}\right)} H_{1}^{(2)}\left(\xi^{A} r\right)\right] .
\end{aligned}
$$

An important characteristic of PWAS, which distinguishes them from conventional ultrasonic transducers, is their capability of tuning into various guided wave modes. A comprehensive study of these prediction formulae in comparison with experimental results has recently been performed by Santoni et al. (2007). Simulation plot of Equation (17) is presented in Figure 6(a) using a $7 \mathrm{~mm}$ square PWAS installed on $1.07 \mathrm{~mm}$ thick 2024-T3 aluminum alloy plate. Note the efficient PWAS length for a $7 \mathrm{~mm}$ PWAS has been verified as $6.4 \mathrm{~mm}$ (Santoni et al., 2007). Equation (17) contains the $\sin (\xi a)$ behavior that displays maxima when the PWAS length $l_{a}=2 a$ equals an odd multiple of the half wavelength, and minima when it equals an even multiple of the half wavelength. A complex pattern of such maxima and minima emerges, since several Lamb modes, each with its own different wavelength, coexist at the same time. The plot in Figure 6(a) shows that at $210 \mathrm{kHz}$, the amplitude of the $\mathrm{A}_{0}$ mode goes through zero, while that of the $\mathrm{S}_{0}$ is close to its peak. This represents an excitation 'sweet spot' for $\mathrm{S}_{0}$ Lamb waves. Experimental results confirming this prediction are presented in Figure 6(b).

\section{PWAS NDE/SHM APPLICATIONS}

In SHM and NDE, PWAS can be used for E/M impedance measurement and, like conventional ultrasonic transducers, can operate in pitch-catch, pulse-echo, and phased-array modes for crack detection in metallic structures.

\section{PWAS E/M Impedance Measurement}

PWAS and the associated structural dynamics identification methodology based on the $\mathrm{E} / \mathrm{M}$ impedance response are ideally suited for small machinery parts that have natural frequencies in the $\mathrm{kHz}$ range (Giurgiutiu and Zagrai, 2005). PWAS are able to detect subtle changes in the high-frequency structural dynamics at local scales. Such local changes in the high-frequency structural dynamics are associated with the presence of incipient damage, which would not be detected by conventional modal analysis sensors that operate at low frequencies. Systematic experiments were performed on circular plates [Figure 7(a)] to assess the crack detection capabilities of this method. As shown schematically in Figure 7(b), five damage groups were considered: one group consisted of pristine plates (Group 0) and four groups consisted of plates with simulated cracks placed at increasing distance from the plate edge (Group 1-4). Each group contained five nominally 'identical' specimens. Thus, the statistical spread within each group could also be assessed. In our study, a $10 \mathrm{~mm}$ circumferential slit was used to simulate an in-service crack. The simulated crack was placed at a decreasing distance from the plate edge. The radial positions 40, 25, 10, and $3 \mathrm{~mm}$ from the sensor were considered.

The experiments were conducted over three frequency bands: $10-40,10-150$, and $300-450 \mathrm{kHz}$. The data was processed by plotting the real part of the $\mathrm{E} / \mathrm{M}$ impedance spectrum, and determining a damage metric to quantify the difference between spectra. The data for the $10-40 \mathrm{kHz}$ band are shown in Figure 8. As damage is
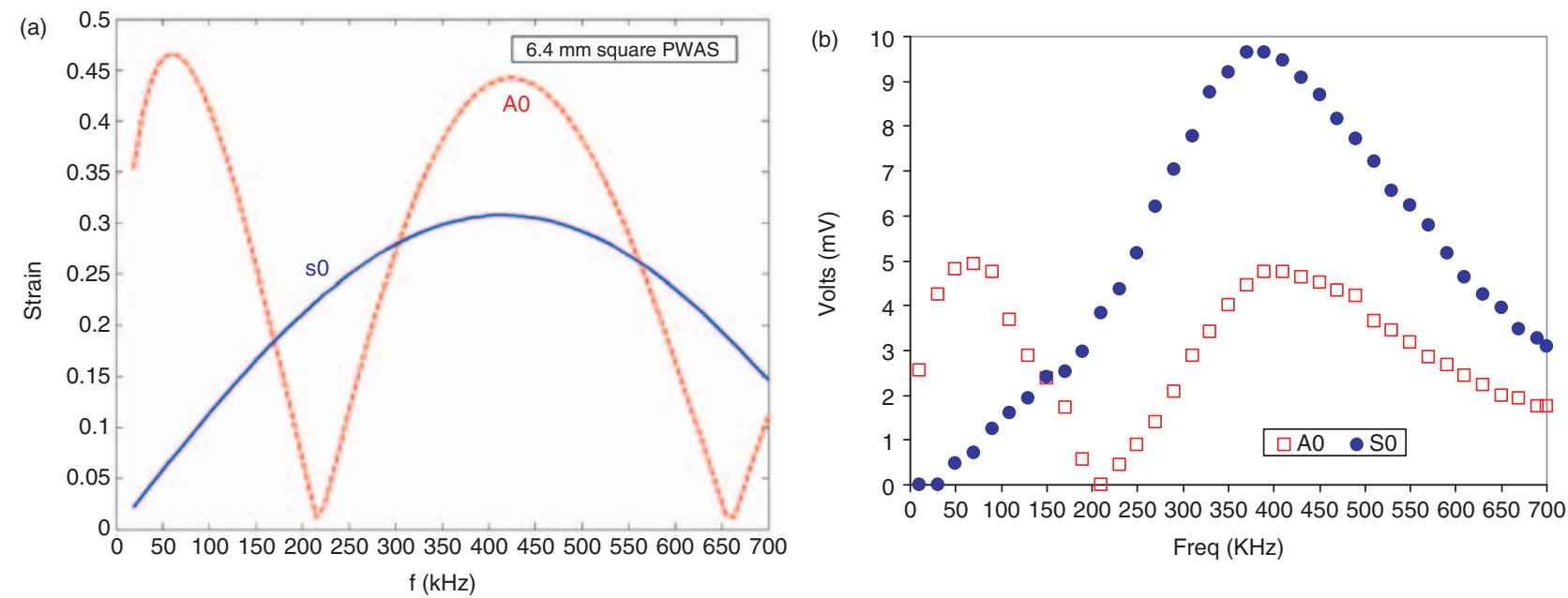

Figure 6. PWAS Lamb wave tuning using a $7 \mathrm{~mm}$ square PWAS placed on $1.07 \mathrm{~mm}$ 2024-T3 aluminum alloy plate: (a) prediction with Equation (17) for $6.4 \mathrm{~mm}$ effective sensor length; (b) experimental results (Santoni et al., 2007). 
introduced in the plate, resonant frequency shifts, peaks split, and the appearance of new resonances are noticed. As the damage becomes more severe, these changes become more profound. The most profound changes are noticed for Group 4. For the higher frequency bands, similar behavior was observed. E/M impedance has also been successfully employed for damage detection in combination with statistic damage metrics (Giurgiutiu and Zagrai, 2005) and probabilistic neural network (PNN) (Specht, 1990).

\section{Ultrasonic NDE/SHM}

Research has been conducted to correlate pitch-catch transmission of elastic waves with varying levels of fatigue damage in the Arcan specimen. The specimen was made out of a $1.2 \mathrm{~mm}$ thick galvanized mild steel sheet and was designed for mixed mode I/II fracture testing with a fixture (Giurgiutiu et al., 2006b).
It was instrumented with nine $7 \mathrm{~mm}$ circular PWAS on one side of the specimen, as shown in Figure 9. Care had been given to keep the PWAS away from the expected crack path. In the pitch-catch tests, a 3-count toneburst signal at a frequency of $474 \mathrm{kHz}$ with $10 \mathrm{~V}$ magnitude was generated by the HP33120 function generator sending out from one PWAS. The receiving signal at another PWAS was recorded with the Trektronix TDS 210 digital oscilloscope. The data was recorded after each stage such that the crack growth data from the images and those from the PWAS could be compared. The tests were done in 12 crack growth stages and damage was evaluated by the RMSD DI analysis. Comparing the DI curves using RMSD method, we found that RMSD works well for monitoring Arcan specimen crack growth under loading. When the crack is small, the reflection of waveforms from the transmitter is increasing with the growth of crack. A linear relationship between damage index (DI) and crack size (a)

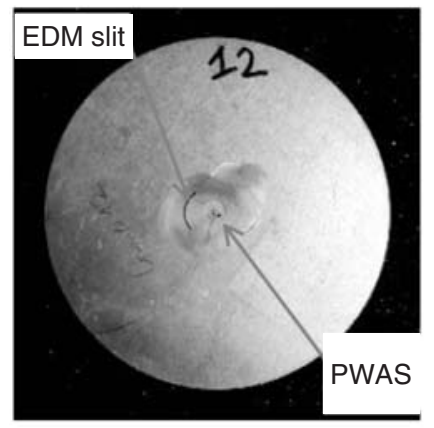

(b)

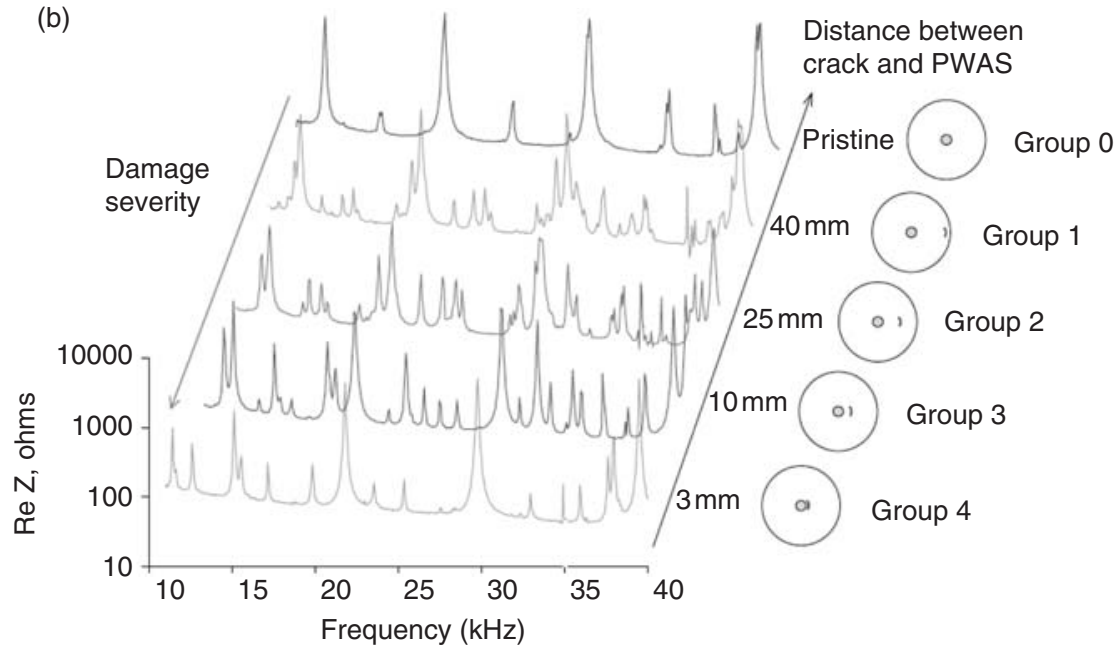

Figure 7. Experiments on dependence of the $E / M$ impedance spectra on the location of damage on metallic plate specimen, E/M impedance in $0.5-40 \mathrm{kHz}$ frequency range: (a) the aluminum plate specimen with PWAS installed in the center; (b) E/M impedance spectra at various crack situations (Giurgiutiu and Zagrai, 2005).

(a)

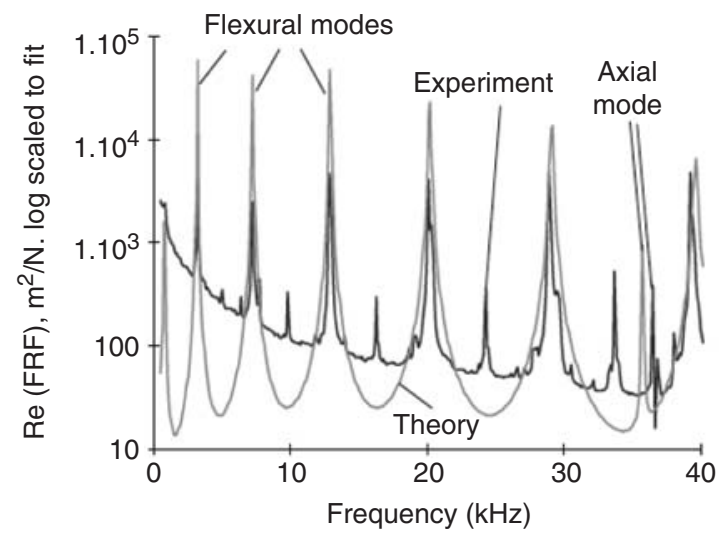

(b)

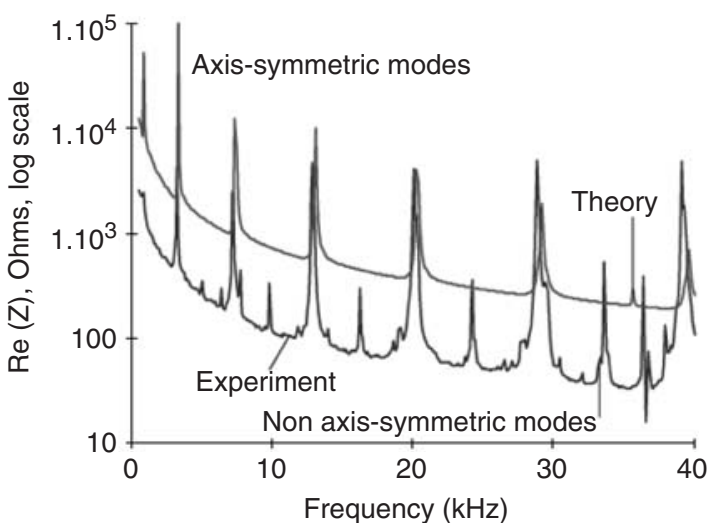

Figure 8. Experimental and calculated spectra for pristine plate specimen: (a) FRF in $0.5-40 \mathrm{kHz}$ range; (b) E/M impedance in 0.5-40 kHz range (Giurgiutiu and Zagrati, 2005). 
(a)

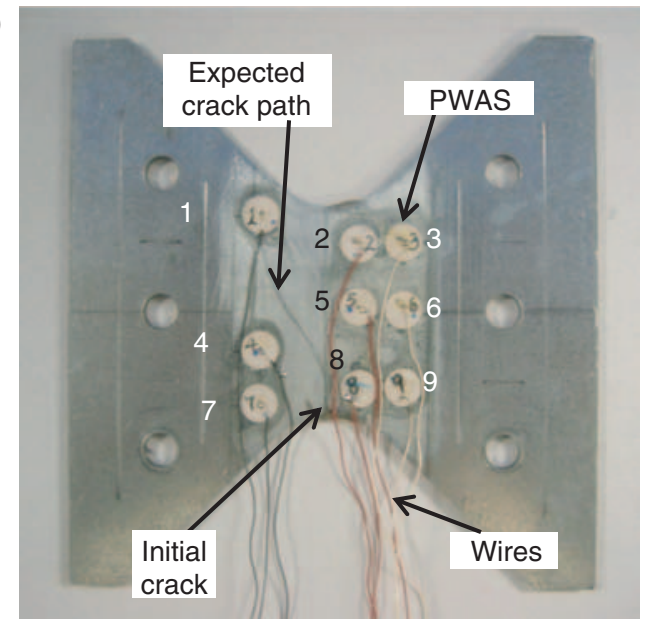

(b)

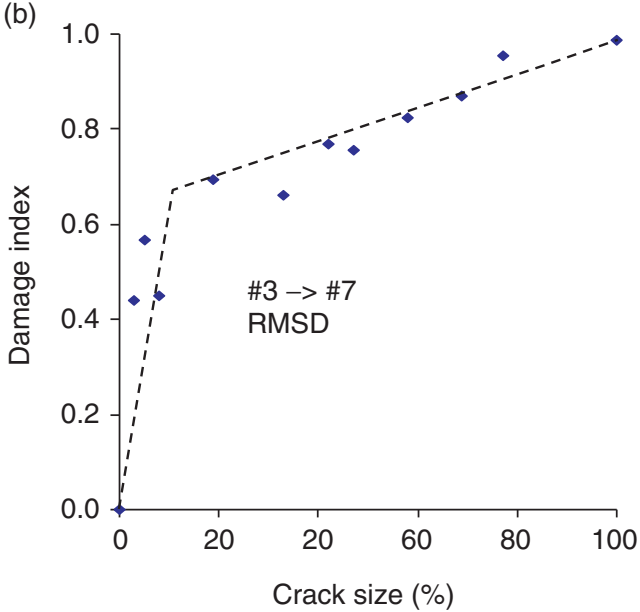

Figure 9. Arcan specimen pitch-catch test. (a) Arcan specimen; (b) RMSD DI curve from PWAS \#3 to \#7 (Giurgiutiu ett al., 2006).

can be perceived. With further growth of crack, the received waveforms are mainly scattered waves.

When guided wave pulse-echo method is used, wide coverage could be achieved from a single location. The wave sent by the PWAS is partially reflected at the crack while the echo is captured at the same PWAS acting as receiver. By comparing the pristine signal and the measurement, difference will indicate the presence and extent of the structural damage. A wave propagation experiment has been done on an aircraft panel to illustrate crack detection (Giurgiutiu et al., 2003b). However, to ensure the crack detection, an appropriate Lamb wave mode must be selected. It has been verified that the $\mathrm{S}_{0}$ Lamb waves can give much better reflections from the through-the-thickness cracks than the $\mathrm{A}_{0}$ Lamb waves experimentally and theoretically (Giurgiutiu et al., 2003a). The selection of such a wave is achieved through the Lamb wave tuning.

PWAS can also be wired as phased arrays to detect damage on thin-wall structures. PWAS phased arrays have been developed for thin-wall structures (e.g., aircraft shells, storage tanks, large pipes, etc.) that use Lamb waves to cover a large surface area through beam steering from a central location. The embedded ultrasonic structural radar (EUSR) algorithm is a method and device for performing ultrasonic damage detection using PWAS phased array for the transmission and reception of guided waves and a signal post-processing methodology based on the beam-forming process. Its principle of operation is derived from two general concepts:

1. The generation of tuned guided Lamb waves with PWAS.

2. The principles of conventional phased-array radar.

As used herein, the term 'embedded' is intended to mean that the transducers are permanently attached directly to the surface of a thin-wall structure (such as using a suitable adhesive). One distinctive feature of this methodology is the provision of an operative mode in which very little instrumentation and minimal power are required to implement the phased array principles compared with the conventional ultrasonic phased array. In EUSR, excitation is implemented on only one PWAS at a time and a round-robin procedure is applied to measure a set of primitive signals that are stored in digital format and processed in virtual time after the data collection. This feature is specific to structural health monitoring implementations which allow for extensive signal processing over time. This feature permits inexpensive and light weight implementation of phased-array beam-forming by avoiding complex multiplexing electronics. Other features and full development of the EUSR algorithm can be found in greater detail in Giurgiutiu and Bao (2004), Yu and Giurgiutiu (2007a,b), and Giurgiutiu et al. (2006a).

\section{PWAS MULTI-MODE CORROSION DETECTION}

PWAS have shown that they are able to perform $\mathrm{SHM} / \mathrm{NDE}$ as either $\mathrm{E} / \mathrm{M}$ impedance spectrum measurement or ultrasonic transducers. In this section, we developed a multi-mode sensing methodology for corrosion detection on metallic structures utilizing a single PWAS network. The PWAS network will operate as ultrasonic transducer, $\mathrm{E} / \mathrm{M}$ impedance sensor, and thickness gauge as well, in order to detect and quantify corrosion damage simply by changing the driving frequencies of PWAS. The multi-mode sensing takes advantage of PWAS abilities: (1) to serve as guided wave transducer, (2) to measure structural impedance spectrum, and (3) to directly measure the wall thickness. The detection procedure consists of three steps:

1. Lamb waves inspection using pitch-catch method. The data will be analyzed by a DI method. The pitch-catch 
(a)

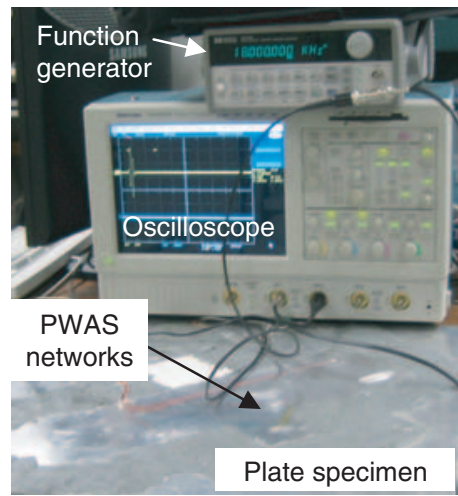

(b)

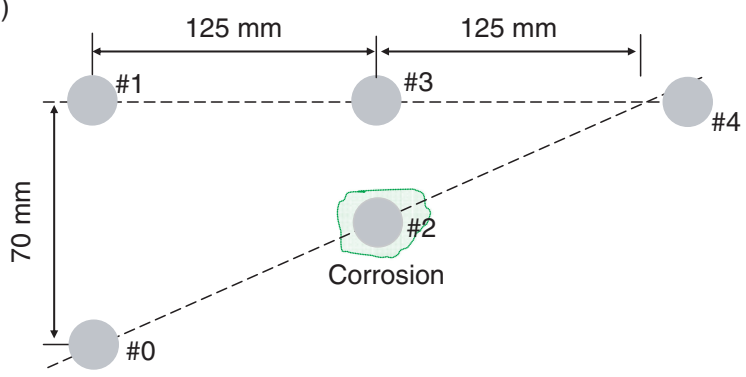

Figure 10. PWAS multi-mode sensing for corrosion detection: (a) experiment set-up for plate-like structure, equipped with function generator and oscilloscope; (b) schematic of a network using five $7 \mathrm{~mm}$-round PWAS.

pair that is suffering the largest change will be considered as having corrosion on that path.

2. Once the path is determined, $\mathrm{E} / \mathrm{M}$ impedance spectrum will be obtained for all the PWAS along the path and analyzed by DI method. The transducer which has the largest change will be determined as the one on the corrosion.

3. Finally, direct thickness measurements will be conducted to quantify the thinning.

By using this strategy, the corrosion area will be located and measured.

In guided wave pitch-catch method, if the wave travels through a region where there is a change in material properties, such as thickness decreasing in corrosion, the directly transmitted signal will be modified and the receiving signal will provide good information about the corrosion development across the transmitting and receiving transducer path. The change can be obtained by subtracting the baseline data recorded for the structure without corrosion from the receiving data for the structure with the developing corrosion damage. The difference is a good indicator for the corrosion detection parameter, since it carries information of both amplitude changes and the phased changes from the corrosion growth. The DI is defined as the relative ratio of the difference between each measurement and baseline signals to the baseline signal:

$$
\mathrm{DI}=\sqrt{\frac{\sum_{j=0}^{N-1}\left[s_{i}(j)-s_{0}(j)\right]^{2}}{\sum_{j=0}^{N-1} s_{0}^{2}(j)}}
$$

where, $s_{i}$ is the $i$-th measurement and $s_{0}$ is the baseline signal, and $N$ is the data length.

\section{Corrosion Detection on Plate Specimen}

The multi-sensing system has been successfully used on a $500 \times 500 \times 3.229 \mathrm{~mm}^{3}$ rectangular aluminum plate
Table 1. Corrosion depth changes.

\begin{tabular}{lcc}
\hline Records & $\Delta \boldsymbol{d}_{\boldsymbol{i}}(\mathbf{m m})$ & $\Delta \boldsymbol{d}_{\boldsymbol{i}} / \boldsymbol{d}_{\mathbf{0}}(\%)$ \\
\hline 0 & 0 & 0 \\
1 & 0.381 & 11.81 \\
2 & 0.7366 & 22.81 \\
3 & 0.9398 & 29.1 \\
4 & 1.09728 & 33.98 \\
5 & 1.27 & 39.33 \\
\hline$d_{0}$ original thickness; $\Delta d_{i}$ removed thickness
\end{tabular}

with the 5-PWAS network, detecting corrosion damage simulated by removing materials from a $5 \times 3.8 \mathrm{~mm}^{2}$ area, as illustrated in Figure 10(a). A five $7 \mathrm{~mm}$-round PWAS network was installed while a corrosion defect located underneath PWAS \#2 is to be added by materials loss through chemical corrosion at room temperature (Figure 10(b)).

Depth changes are given in the Table 1. Lamb wave tuning finds the frequency at $57 \mathrm{kHz}$, where $\mathrm{A}_{0}$ mode dominates while $\mathrm{S}_{0}$ mode is minimized.

\section{PITCH-CATCH TESTS}

Pitch-catch experiments were conducted first, sending out a 3-count tone-burst signal from a HP 33120A signal generator to one PWAS and the receiving signal at another PWAS, which is recorded by a TDS-210 digital oscilloscope. For Lamb waves, two basic modes, $\mathrm{A}_{0}$ and $\mathrm{S}_{0}$, co-exist at the low $f d$ production, such as $120 \mathrm{kHz}$. As signals shown in Figure 11(a), from PWAS $\# 0$ to $\# 4, S_{0}$ travels faster and arrives earlier but with much smaller amplitude than $\mathrm{A}_{0}$ mode. DI curves on each individual mode (wave packet), as given in Figure 11(b), showed that $S_{0}$ mode did not change significantly with the corrosion development which confirmed itself as a less sensitive mode to corrosion damage (Giurgiutiu et al., 2003a).

Using PWAS Lamb wave tuning technique, dominant $\mathrm{A}_{0}$ was obtained at the $57 \mathrm{kHz}$ excitation frequency. Two sets of signals were collected at PWAS \#4 when PWAS \#0 sent (as illustrated in 
Figure 11(c)) and when PWAS \#1 sent, respectively. The DI analysis was then applied to the measurements to achieve an evaluation of the health of the plate. The resulting DI curves are shown in Figure 11(d). For the DI of PWAS pair 1-4, since the corrosion is away from the path, the arriving wave packet is less affected by the growth of corrosion as that of pair $0-4$.
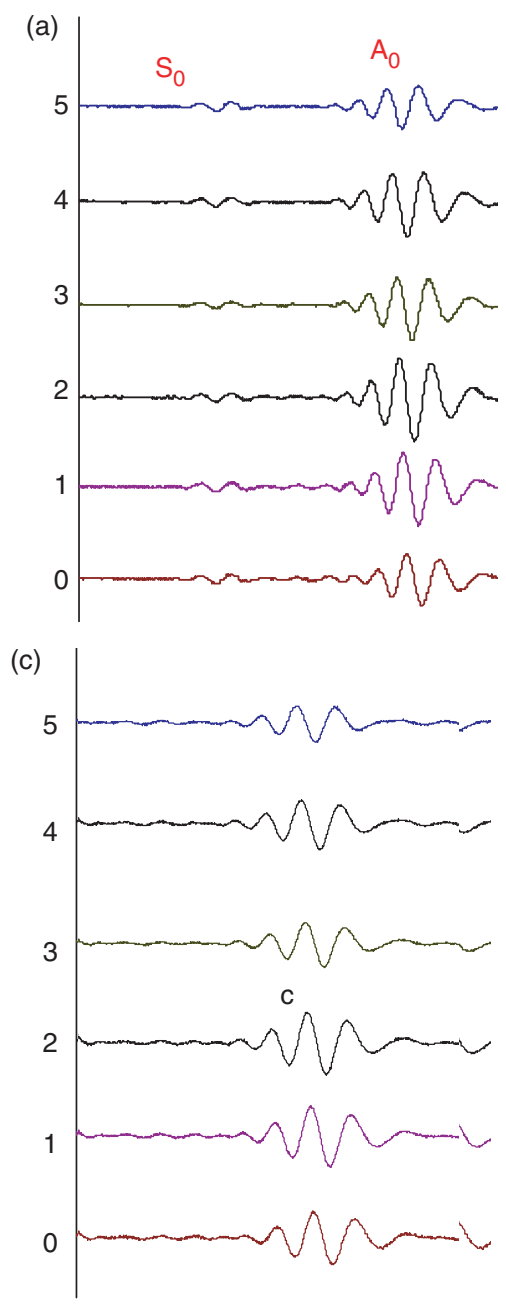

(b)

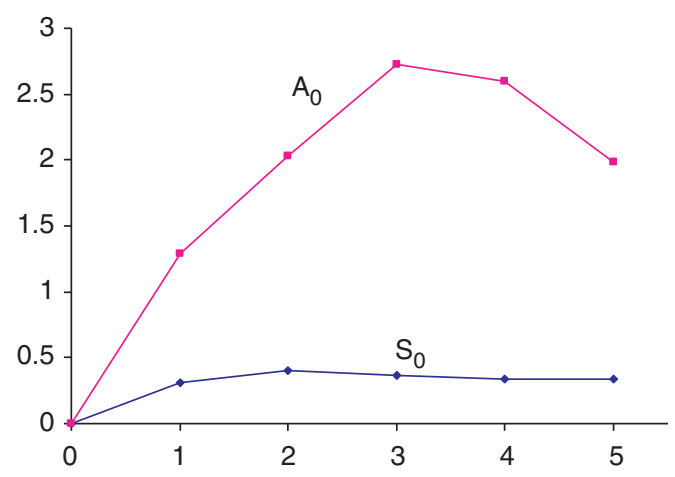

(d)

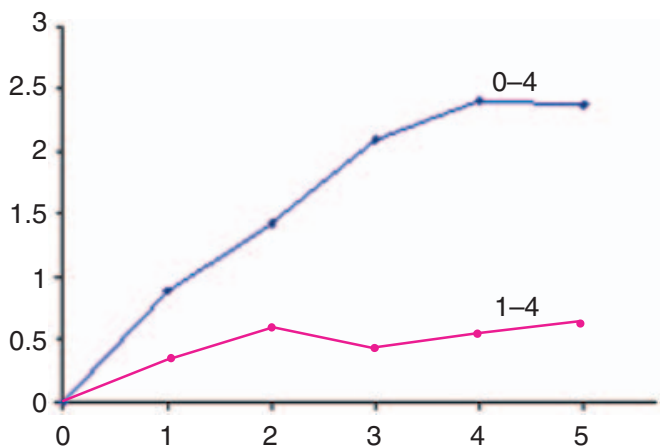

Figure 11. Pitch-catch DI analysis for corrosion detection on metallic plate: (a) received signals at PWAS \#4 when PWAS \#0 sent at $120 \mathrm{kHz}$; (b) DI curves for $A_{O}$ and $S_{0}$ mode; (c) received signals at PWAS \#4 when PWAS \#0 sent at $57 \mathrm{kHz}$; (d) DI curves for PWAS pair 0-4 and 1-4.

(a)

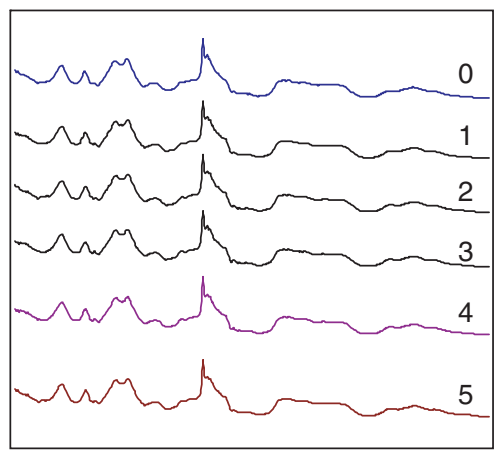

(b)

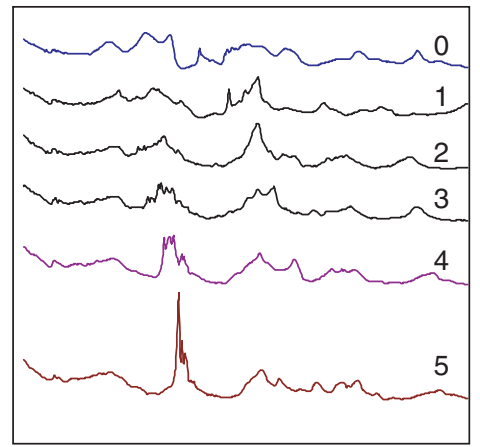

(c)

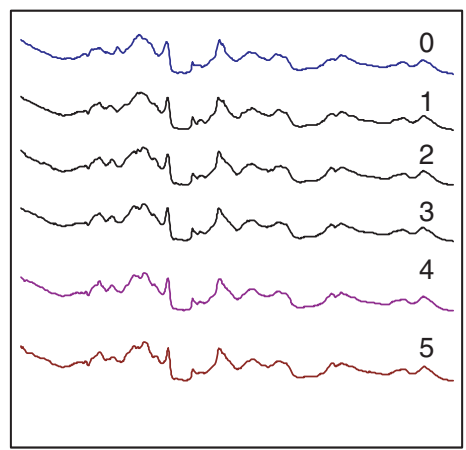

Figure 12. E/M impedance spectra at PWAS \#0, \#2, and \#3 at various corrosion stages in the band 200-2000 kHz: (a) PWAS \#0; (b) PWAS \#2; (c) PWAS \#3. 
symmetric with \#0 about \#2, and \#3 was intentionally investigated in order to show the difference between \#2 and \#3.) The spectrum difference between 'baseline' data and 'corroded' data can be quantified by processing the data, the real part of the $\mathrm{E} / \mathrm{M}$ impedance spectra, with DI method. The DI curves of the spectra for PWAS \#0, \#2, and \#3 are shown in Figure 13.

\section{PWAS THICKNESS MEASUREMENT}

Although PWAS have been widely used as an ultrasonic transducer operating in the $d_{31}$ mode (inplane motion), its potential as a thickness gauge in $d_{33}$ mode (out-of-plane motion) has not been fully explored (Giurgiutiu and Lyshevski, 2003). A proof-of-concept investigation was first performed to verify that PWAS can indeed be used for thickness measurement. Figure 14(a) shows the actual experimental set-up. The system consists of a plate specimen with a square or round PWAS attached to one surface of the plate. The PWAS are connected with thin insulated wires to a signal bus that ends at a digital pulse/echo device. Under electric excitation, the PWAS generates an elastic Lamb wave pulse that spreads out into the plate following a thickness wave pattern. When the wave pulse hits the other surface, it is reflected back as an echo to the PWAS.

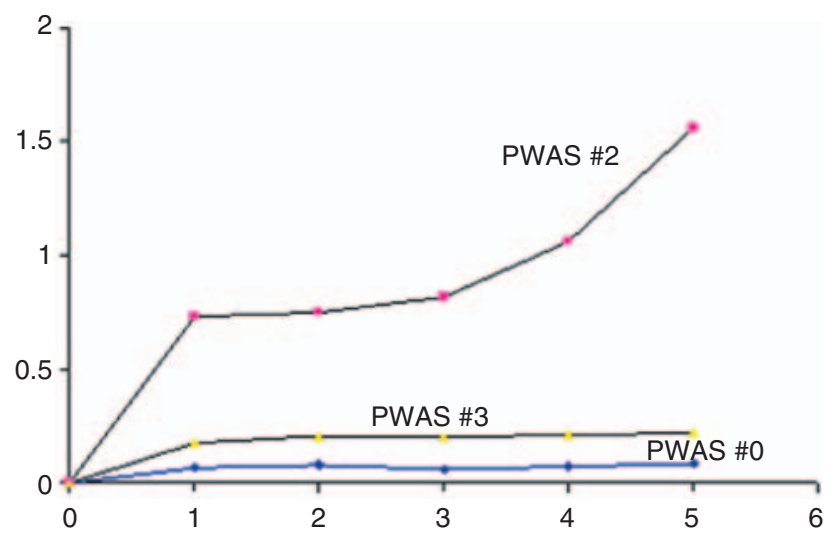

Figure 13. E/M impedance spectra DI curves of PWAS \#0, \#2, and \#3.

(a)

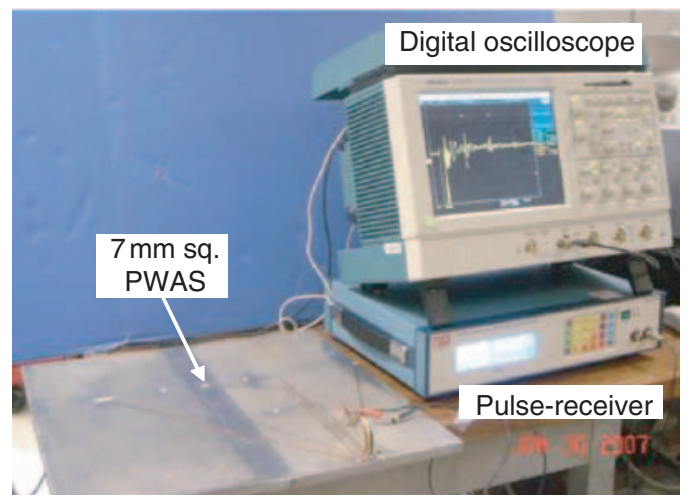

The elastic Lamb waves are transmitted back as an electric signal that is then picked up by the digital oscilloscope synchronized with the pulse/echo device. This set-up is equivalent to the measurement of the time of flight of the reflective echo for the calculation of plate thickness. A typical pulse-echo signal is shown in Figure 14, which indicates a delay of $\Delta t$ between the initial pulse and the first reflected echo. The echo strength is strongest at first, which is the first reflected wave from the lower surface, and becomes weaker for the later echoes.

Given the wave speed $c$ of the specified material, the thickness $T$ can be calculated as:

$$
T=\frac{c \cdot \Delta t}{2} .
$$

In the plate corrosion detection experiment, once the sensor undergoing most significant impedance change was identified, i.e., PWAS \#2, it was used to directly measure the thickness at its position. In order to evaluate the performance of PWAS thickness measurement, a $30 \mathrm{MHz}$ conventional ultrasonic transducer has also been used in parallel for comparison. The measurement results are given in Table 2 .

It shows that PWAS provides reasonable results despite their low cost. Subsequent work is needed to investigate error ranges of both conventional transducers and PWAS. A high-performance thickness gauge is

Table 2. Thickness measurement using PWAS \#2 and conventional ultrasonic transducer.

\begin{tabular}{llllll}
\hline Records & $\begin{array}{c}\boldsymbol{T}_{\mathbf{0}} \\
\mathbf{( m m )}\end{array}$ & $\begin{array}{c}\boldsymbol{T}_{\text {PWAS }} \\
\mathbf{( m m )}\end{array}$ & $\begin{array}{c}\boldsymbol{\varepsilon}_{\text {PWAS }} \\
\mathbf{( \% )}\end{array}$ & $\begin{array}{c}\boldsymbol{T}_{\text {ultrasonic }} \\
\mathbf{( m m )}\end{array}$ & $\begin{array}{c}\boldsymbol{\varepsilon}_{\text {ultrasonic }} \\
\mathbf{( \% )}\end{array}$ \\
\hline 0 & 3.229 & 3.354 & 3.87 & 3.069 & 4.96 \\
1 & 2.848 & 2.9072 & 2.08 & 2.7022 & 5.12 \\
2 & 2.4924 & 2.5034 & 4.41 & 2.3481 & 5.78 \\
3 & 2.2892 & 2.298 & 3.84 & 2.174 & 5.03 \\
4 & 2.137 & 2.218 & 3.79 & 2.062 & 3.50 \\
5 & 1.959 & 2.1742 & 10.98 & 1.839 & 6.13 \\
\hline$T_{0}$-theoretical thickness, c-wave velocity, $6.212 \mathrm{~mm} / \mu \mathrm{s}$. &
\end{tabular}

(b)

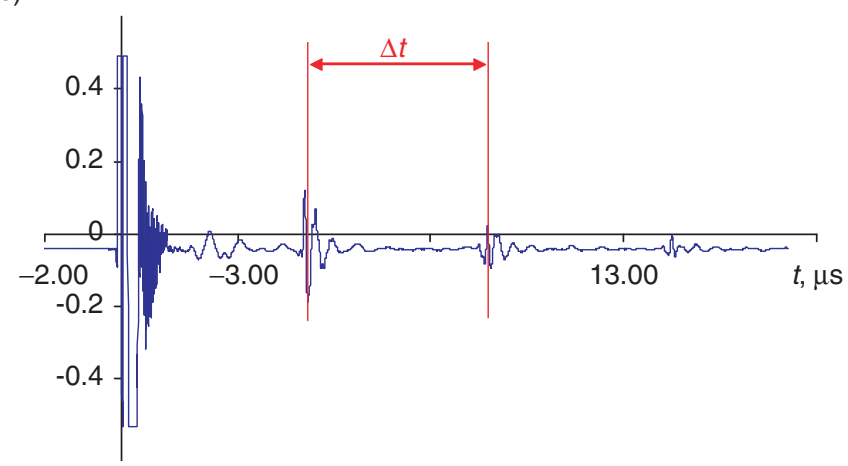

Figure 14. PWAS thickness measurement: (a) laboratory experiment set-up using oscilloscope and pulse-receiver; (b) a pulse-echo signal captured by the $7 \mathrm{~mm}$-round PWAS installed on the $13.5 \mathrm{~mm}$ steel plate. 
(a)

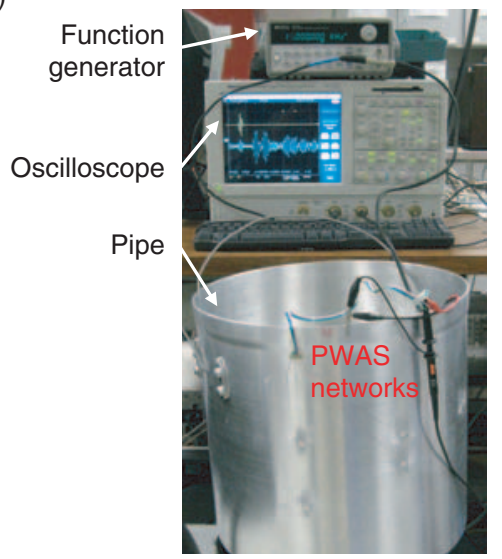

(b)

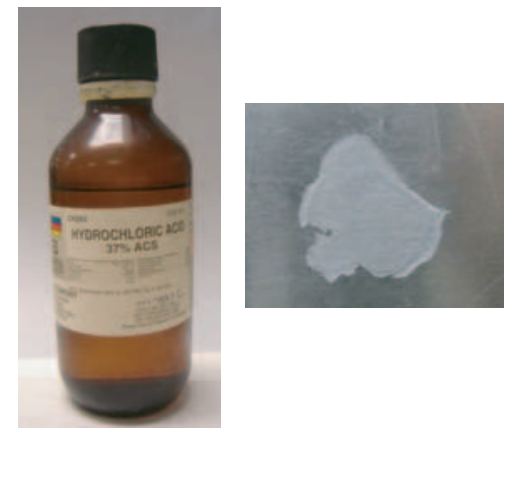

Figure 15. Multi-mode sensing for corrosion detection in pipe specimen: (a) pipe test set-up; (b) chemical etching.

(a)

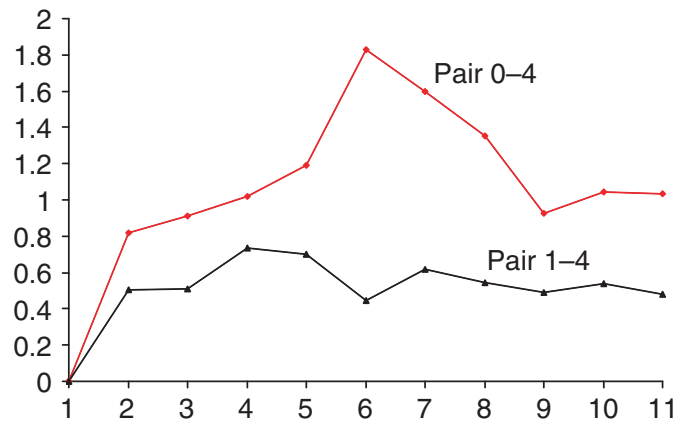

(b)

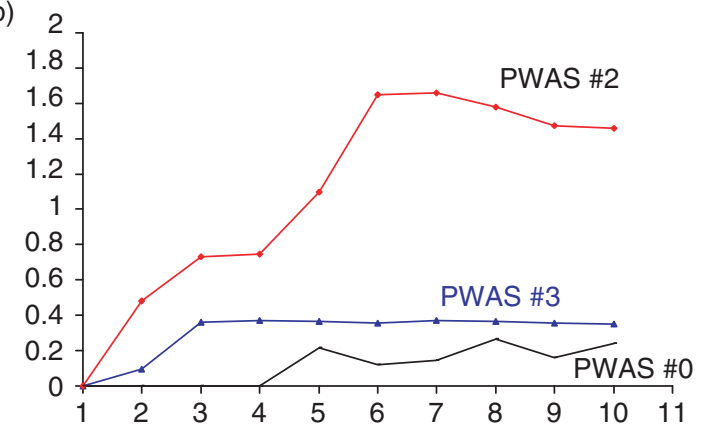

Figure 16. DI analysis for corrosion detection on pipe specimen: (a) DI curves for pitch-catch tests at $57 \mathrm{kHz}$ on PWAS pairs $0-4$ and 1-4; (b) DI curves for impedance measurements on PWAS \#0, \#2, and \#3.

to be considered and further investigation on the error ranges of the two transducers is to be conducted.

\section{Corrosion Detection in Pipe Structure}

The multi-mode sensing has also been tested in a pipe specimen (Figure 15(a)). The same 5-PWAS network was installed on a $1.67 \mathrm{~mm}$ thick aluminum pipe, with corrosion simulated through chemical etching (hydrochloric acid 37\% as shown in Figure 15(b); the depth was controlled via etching time rather than precise depth measurement). RMSD DI analysis results are given in Figure 16 for pitch-catch at $57 \mathrm{kHz}$ and impedance measurements in $200-2000 \mathrm{kHz}$ (the first three points in PWAS \#0 impedance measurement were not applicable). The comparison of pitch-catch DI curves shows that pair 0-4 was undergoing the corrosion while comparison of impedance DI shows that PWAS \#2 is the corrosion position. Note that we are unable to obtain meaningful thickness readings in the pipe tests since the pipe wall thickness is smaller than the smallest readable value in Table 2.

\section{CONCLUSIONS}

In this paper, a SHM/NDE technology using PWAS was presented. The PWAS transducer can be permanently attached onto the structures and operate in $\mathrm{E} / \mathrm{M}$ impedance mode and ultrasonic waves mode using pitch-catch and pulse-echo methods. We gave the principles of PWAS using 1D and 2D models and explained how they work as impedance measurement devices and as ultrasonic transducers to generate and receive propagating guided waves in thin-wall structures, i.e., Lamb waves. Particularly in ultrasonic applications, the Lamb wave tuning was introduced, which enables the use of embedded PWAS for SHM or NDE.

Several application examples were presented to show the multi-mode sensing abilities with PWAS for in situ damage detection in thin-wall structures. They show that PWAS can be used for E/M impedance measurement, in pitch-catch mode for crack growth monitoring, and for constructing phased-arrays, respectively. However, in the integrated multi-mode sensing system designed for corrosion detection, we are able to employ several methods altogether using a sensor network simply by changing PWAS operational frequencies. The multimode sensing uses the $\mathrm{A}_{0}$ Lamb wave mode to detect structural changes along the pitch-catch pair in combination with DI analysis. This analysis yields the path that is undergoing corrosion damage. Then the impedance spectra of all the PWAS on the identified sensor path are measured to find out more localized information, i.e., which PWAS is closest to the corrosion. Finally, after the 
location is identified, this PWAS is used as a thickness gauge to quantify the changes in thickness. Experimental results on both aluminum plate and pipe structures yielded good detection results and verified the feasibility of the multi-mode sensing.

Although remarkable progress has been made with multiple modes PWAS sensing, considerable work remains to be done. To increase the acceptance of this emerging technology, the refining of the theoretical analysis and calibration against well-established experiments is needed, especially for applications in thickness mode. In addition, the operational and environmental variations of the monitored structure need to be addressed. It shows signals will change due to varying operational and environmental conditions of the structure such as temperature, humidity, etc. Also, the bonding layer between the PWAS and structure is often the durability weak link for SHM applications, leading to loss of contact with the structure. The bonding layer may also include acoustic impedance mismatch with detrimental effects on damage detection. Better durability may therefore be expected from a builtin sensor that is incorporated into the structure, such as the in situ fabricated PWAS. Last, but not least, a chiplevel PWAS device possessing local processing ability and wireless communication ability must be developed to achieve in-field in situ SHM applications.

\section{ACKNOWLEDGMENTS}

Financial support from the National Science Foundation, grants NSF CMS-0408578 and NSF CMS-0528873, and Air Force Office of Scientific Research grant FA9550-04-0085 are gratefully acknowledged.

\section{REFERENCES}

Alleyne, D.N. and Cawley, P. 1992. "Optimization of Lamb Wave Inspection Techniques,” NDT International, 25(1):11-22.

Crawley, E.F. and de Luis, J. 1987. "Use of Piezoelectric Actuators as Elements of Intelligent Structures," AIAA Journal, 25:1373-1385.

Giurgiutiu, V. 2005. "Tuned Lamb-wave Excitation and Detection with Piezoelectric Wafer Active Sensors for Structural Health Monitoring," Journal of Intelligent Material Systems and Structures, 16(4):291-306.

Giurgiutiu, V. and Bao, J. 2004. "Embedded-ultrasonics Structural Radar for in-situ Structural Health Monitoring of Thin-wall Structures," Structural Health Monitoring - An International Journal, 3(2):121-140.
Giurgiutiu, V. and Cuc, A. 2005. "Embedded NDE for Structural Health Monitoring, Damage Detection, and Failure Prevention," Shock and Vibration Reviews, 37(2):83-105.

Giurgiutiu, V. and Lyshevski, S.E. 2003. Micromechatronics: Modeling, Analysis, and Design with MATLAB, CRC Press LLC, Florida.

Giurgiutiu, V. and Zagrai, A. 2005. "Damage Detection in Thin Plates and Aerospace Structures with the Electro-mechanical Impedance Method," Journal of Structural Health Monitoring, 4(2):99-1183.

Giurgiutiu, V., Bao, J. and Zagrai, A.N. 2006a. "Structural Health Monitoring System Utilizing Guided Lamb Waves Embedded Ultrasonic Structural Radar," Patent No. US 6,996,480 B2, Date of Patent Feb. 7.

Giurgiutiu, V., Bao, J. and Zhao, W. 2003a. "Piezoelectric-Wafer Active-Sensor Embedded Ultrasonics in Beams and Plates," Experimental Mechanics, 43(4):428-449.

Giurgiutiu, V., Xu, B., Chao, Y., Liu, S. and Gaddam, R. 2006b. "Smart Sensors for Monitoring Crack Growth under Fatigue Loading Conditions," Journal of Smart Structures and Systems, 2(2):101-113.

Giurgiutiu, V., Zagrai, A.N., Bao, J., Redmond, J., Roach, D. and Rackow, K. 2003b. "Active Sensors for Health Monitoring of Aging Aerospace Structures," International Journal of the Condition Monitoring and Diagnostic Engineering Management, 6(1):3-21

Han, J., Kim, C.G. and Kim, J.Y. 2006. "The Propagation of Lamb Waves in a Laminated Composite Plate with a Variable Stepped Thickness," Journal of Composite Structures, 76(4):388-396

Ihn, J.B. and Chang, F.K. 2002. "Built-in Diagnostics for Monitoring Crack Growth in Aircraft Structures," In: Proceedings of the SPIE 9th International Symposium on Smart Structures and Materials, 17-21 March, San Diego, CA, paper \# 4702-04.

Lin, X. and Yuan, F.G. 2001. "Diagnostic Lamb Waves in an Integrated Piezoelectric Sensor/Actuator Plate: Analytical and Experimental Studies," Smart Materials and Structures, 10:907-913

Nieuwenhuis, J.H., Neumann, J.J., Greve, D.W. and Oppenheim, I.J. 2005. "Generation and Detection of Guided Waves using PZT Wafer Transducers," IEEE Transactions on Ultrasonics, Ferroelectronics, and Frequency Control, 52(11):2103-2111.

Raghavan, A. and Cesnik, C.E.S. 2005. "Finite-dimensional Piezoelectric Transducer Modeling for Guided Wave Based Structural Health Monitoring," Journal of Smart Materials and Structures, 14:1448-1461

Rose, J.L. 1999. Ultrasonic Waves in Solid Media, Cambridge University Press, New York.

Rose, J.L., Pelts, S.P. and Quarry, M.J. 1998. "A Comb Transducer Model for Guided Wave NDE," Ultrasonics, 36:163-169.

Santoni, G.B., Yu, L., Xu, B. and Giurgiutiu, V. 2007. "Lamb Wave Mode Tuning of Piezoelectric Wafer Active Sensors for Structural Health Monitoring," Journal of Vibration and Acoustics, 129(6):752-762.

Specht, D.F. 1990. "Probabilistic Neural Networks," Neural Networks, 3:109-118.

Yu, L. and Giurgiutiu, V. 2007a. "In-situ 2-D Phased Arrays Guidedwave Damage Detection with Piezoelectric Wafer Active Sensors," Journal of Ultrasonics, 48(2):117-134.

Yu, L. and Giurgiutiu, V. 2007b. "In-situ Optimized PWAS Phased Arrays for Lamb Wave Structural Health Monitoring," Journal of Mechanics of Materials and Structures, 2(3):459-487. 